\title{
Effect of Variable Fluid Properties and Magneto- Hydrodynamics for Convection with Couple Stress Fluid
}

\author{
Nalinakshi Narasappa ${ }^{1}$ (D), Dinesh Pobbathy Ashwathnarayana 2,*(i), \\ Harichandra Boodhgiri Prahlad ${ }^{3}$ (D), Likhith Girish 4 (D)
}

1 Department of Basic Sciences, Atria Institute of Technology (Affiliated to VTU), Bangalore -560024, Karnataka, India; bsehod@atria.edu(N.N.);

2 Department of Mathematics, M S Ramaiah Institute of Technology, Bengaluru - 560 064, Karnataka, India; dineshdpa@msrit.edu (D.P.A);

3 Department of Mechanical Engineering, M S Ramaiah Institute of Technology, Bengaluru - 560 054, India; bpharichandra@msrit.edu (H.B.P.);

4 Department of Mechanical Engineering, JSSATE, Bengaluru, Karnataka, India; likhith784@ gmail.com (L.G.);

* Correspondence: dineshdpa@msrit.edu;

Scopus Author ID 55934627800

Received: 11.01.2021; Revised: 7.02.2021; Accepted: 9.02.2021; Published: 13.02.2021

\begin{abstract}
An attempt is made for analyzing MHD mixed convection over a vertical heated plate with a couple of stress fluid numerically in a systematic manner. Unlike other research-based on literature surveys, the fluid properties are varied here where convection improves drastically compare to fixing them constantly. The boundary layer flow is estimated through mathematically formulated equations for the physical configuration considered. These formulated equations are very tedious to solve with specified boundary conditions in nature. Similarity transformations, RKF scheme, and NR method are used to convert those tedious non-linear PDE to higher-order ODE and hence to first order. Interpretation of various significant parameters is studied and observed their effects with momentum, energy, and solutal equations producing the fluid flow with the plotted graphs. The contribution of the Magnetic field is observed in velocity by reducing the force of the fluid flow. This work's main contribution is to see the effective convection with significant fluid flow parameters, with the inclusion variable fluid properties. $\mathrm{Nu}$ and $\mathrm{Sh}$ numbers are also computed. Certain added effects making them void are well suited and matched with researchers' previous works with a better agreement.
\end{abstract}

Keywords: heat and mass transfer; couple stress fluid; variable fluid properties; porous medium; MHD.

(C) 2021 by the authors. This article is an open-access article distributed under the terms and conditions of the Creative Commons Attribution (CC BY) license (https://creativecommons.org/licenses/by/4.0/).

\section{Introduction}

The fluid flow behavior and its characteristics cannot be exactly described by the conventional non-polar fluids, which are Newtonian fluids. Hence, in the present era, the theory of polar fluids, which are non-Newtonian, takes special attention by many authors and scientists as they require for industrial processes like a solidification of liquid crystals, colloidal and suspension solutions, extrusion of polymer fluids, cooling of the metallic plate and exotic lubrications. Couple stress fluids, which own their distinct features like polar effects and having a magnitude of pressure rise very high, are one among the non-Newtonian fluids with their growing importance in recent technology and industries. The physiological process can happen with magnetic therapy, bearings, and many others. The former can be observed in generators, flow-meters, pumping liquid pumps with the presence of a magnetic field. The latter is the 
bearing - its performance can be improvised by adding the couple stress fluids, which we call additives.

The theory of additives mentioned above and the constructive equations were given by stokes [1]. When the fluids are with very large molecules, there acts a couple of stress fluids in the notable magnitudes. Such large molecules were found in the long-chain hyaluronic acid molecules as additives in synovial fluid, which is modeled as a couple of stress fluid in human joints by Walicki and Walicka [2]. Mechanical interactions in the deforming continuum lead to a couple of stress fluids effects as the large molecules show all the important features. The simplest generalization classical theory here allows the polar effects such as the couple stresses whose theory of fluids defines rotation field in terms of the velocity field.

Couple stress fluid between the parallel plates was studied by Devakar et al. [3], who initially considered for fluid to be incompressible termed as the Stokes' problems. The pattern of florets known as the vogel's model was studied for laminar flow o couple stress fluid by Farooq et al. [4]. Oladeinde et al. [5] analyzed these non-newtonian fluid lubricants' effects on pressure and the load capacity as the slider bearing is exponentially shaped wide infinitely. Further, Natural convection for fluid flow with porous medium varying the permeability in slip flow regime in the presence of additives for mass transfer was analyzed by Nidhish Kumar Mishra et al. [6].

Most of the above-listed literature's research scholars considered non-variation of fluid properties, but the importance of varying them and their enormous usage is not discussed. However, the Investigations have been made varying the permeability by Mohammed \& ElShaer [7]. They considered the physical configuration for convection as the system existing here in this paper. Further, Nalinakshi et al. [8] attempted to study heat and mass transport varying the fluid properties numerically. Nalinakshi et al. [9] continued to see the effect of additive fluids. Later, Suresh Babu et al. [10] continued to study the oscillatory flow. Uma et al. [11] attempted to study the effects of Forchheimer, Magnetohydrodynamics, and radiation absorption for chemically reacting unsteady dusty viscoelastic fluid Couette flow in an irregular channel. Further, the Forchheimer model with MHD over a vertical heated plate was considered and analyzed for thermo-diffusion and diffusion-thermo effects by Nalinakshi et al. [12].

Variation of the fluid properties gradually has been studied by different researchers with different fluids and observed better results and effects on the convection analysis [13-16]. Double Diffusive convection and IHG with soret and dufour effects over an accelerating surface varying the viscosity and permeability was studied by Girinath Reddy et al. [17]. Mixed convective heat transfer, numerical simulation of double-diffusive convection, was also analyzed considering properties' variability [18-20]. Irfan et al. [21] explained the features of nanoparticles mass flux conditions and non-uniform heat sink/source on magnetic Oldroyd-B nano-fluid. Incorporating the energy and mass fluxes caused by mass concentration and temperature gradients were explored by Jawad Ahmed et al. [22] for heat and mass transfer mechanisms. Flow characteristics of free convective nano-fluid flow and an exponentially accelerating vertical plate in the magnetic field's presence and its theoretical analysis were given by Sravan Kumar et al. [23].

Shreedhar et al. [24] examined the two-dimensional, unsteady, laminar, MHD natural convective flow past a vertical plate in the presence of heat absorption and chemical reaction through a porous medium is considered. Unsteady MHD heat and mass transfer over a moveable vertical plate with the influence of thermophoresis and thermal radiation was 
investigated by Ayegbusi Florence et al. [25]. Anantha Kumar Kempannagari et al. [26] examined the convective heat transfer characteristics on magnetohydrodynamic stagnation point flow of micropolar fluid past an exponentially curved surface. Veera Krishna et al. [27] conducted elaborate scrutiny on the Soret and Joule effects of MHD mixed convective flow of an incompressible and electrically conducting viscous fluid past an infinite vertical porous plate taking Hall effects into account. Dinesh et al. [28] examined the effects of Forchheimier and radiation adsorption on MHD double-diffusive unsteady dusty viscoelastic Couette flow in an irregular channel with chemical reaction. Unsteady MHD free convective flow past an inclined plate was analyzed by Shankar Goud et al. [29]. Uma et al. [30] analyzed Forchheimer, Soret, and Dufour's combined effects on free forced convective dusty viscoelastic Couette flow in an irregular channel under a constant magnetic field. Multislip effects on the MHD mixed convection unsteady flow of micropolar nano-fluids over a stretching / shrinking sheet along with radiation in the presence of a heat source was investigated by Sohaib Abdal et al. [31]. Non-linear mixed convective oscillatory flow embedded with a semi-infinite vertical porous plate in the presence of a uniform magnetic field was investigated by Basavaraj et al. [32]. Analytical and numerical study of Soret and Dufour effects on thermosolutal convection in a horizontal Brinkman porous layer with a stress-free upper boundary was investigated by Ismail Filahi et al. [33].

Hence, in continuation, our main motto for the study here is to understand the effect of non-Newtonian, an additive fluid, couple stress fluid with magnetic effect over a vertical heated plate for heat and mass transport process. The main observations are made for fluid properties variation concerning the added fluid and magnetic effect.

\section{Materials and Methods}

\subsection{Mathematical Formulation of the Problem}

The two-dimensional steady flow varying the fluid properties over a heated plate for MHD mixed convective is considered. The additive fluids are sparsely packed. From the leading edge of the plate, the $\mathrm{x}$-coordinate is measured and normally the $\mathrm{y}$-coordinate.

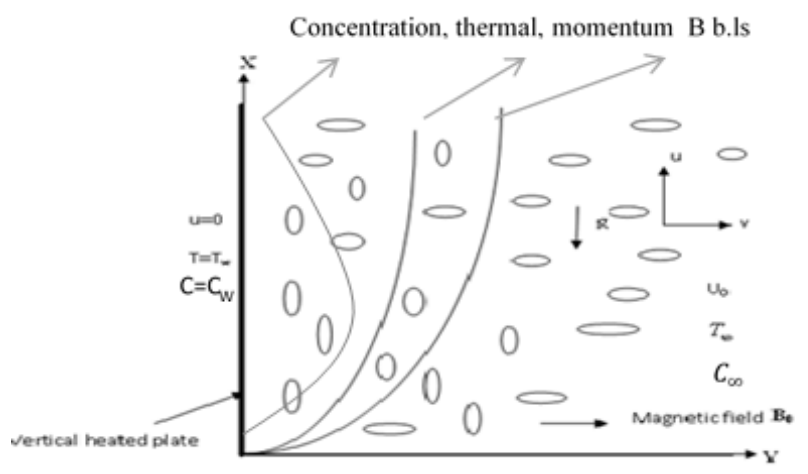

Figure 1. Physical system.

The gravitational force, $\mathrm{g}$, and free stream velocity Uo are in the downward and upward direction, respectively, parallel to the plate. $\mathrm{T}_{\mathrm{w}}$ and $\mathrm{C}_{\mathrm{w}}$ are temperature and concentration of fluid maintained uniformly constant at the plate's surface. They are higher than the values existing in the infinite region away from the plate. Under the Bousinesque approximation being valid, the basic governing equations take the form: 
$\frac{\partial u}{\partial x}+\frac{\partial v}{\partial y}=0$

$u \frac{\partial u}{\partial x}+v \frac{\partial u}{\partial y}=g \beta_{T}\left(T-T_{\infty}\right)-g \beta_{c}\left(c-c_{\infty}\right)+\frac{\bar{\mu}}{\rho} \frac{\partial^{2} u}{\partial y^{2}}-\frac{\mu \varepsilon(y)}{\rho k(y)}\left(U_{o}-u\right)-\frac{\mu_{c}}{\rho} \frac{\partial^{4} u}{\partial y^{4}}-\frac{\sigma_{m} B_{0}^{2}}{\rho} \varepsilon(y) u$,

$u \frac{\partial T}{\partial x}+v \frac{\partial T}{\partial y}=\frac{\partial}{\partial y}\left(\alpha(y) \frac{\partial T}{\partial y}\right)+\frac{\bar{\mu}}{\rho_{o} C_{p}}\left(\frac{\partial u}{\partial y}\right)^{2}$

$u \frac{\partial C}{\partial x}+v \frac{\partial C}{\partial y}=\frac{\partial}{\partial y}\left(\gamma(y) \frac{\partial C}{\partial y}\right)$

under the boundary conditions of the physical system shown below:

$u=0, \quad v=0, \quad v_{x}=u_{y}, \quad T=T_{w}, \quad C=C_{w} \quad$ at $\quad y=0$,

$u=U_{o}, \quad v_{x}=u_{y}, \quad T=T_{\infty}, \quad C=C_{\infty}$ as $y \rightarrow \infty$.

Equations (1) to (4) are highly coupled, very difficult to work for the solution. They are also non-linear in nature. Hence, Similarity transformations, dimensionless variables, stream function with its partial derivative named as $\mathrm{u}$ and $\mathrm{v}$ (the velocity components) satisfying the continuity equation are used, and their expressions are:

$\eta=\left(\frac{y}{x}\right)\left(\frac{U_{o} x}{v}\right)^{1 / 2}, \quad \psi=\sqrt{v U_{o} x} f(\eta), \theta=\frac{T-T_{\infty}}{T_{W}-T_{\infty}}, \phi=\frac{\mathrm{C}-\mathrm{C}_{\infty}}{C_{w}-C_{\infty}}$
$u=U_{o} f^{\prime}(\eta), \quad v=-\frac{1}{2} \sqrt{\frac{v U_{o}}{x}}\left(f(\eta)-\eta f^{\prime}(\eta)\right)$,

As this study emphasizes the fluid properties to be varied, they can be expressed as follows:

$$
\begin{aligned}
& k(\eta)=k_{o}\left(1+d e^{-\eta}\right), \\
& \varepsilon(\eta)=\varepsilon_{o}\left(1+d^{*} e^{-\eta}\right), \\
& \alpha(\eta)=\alpha_{o}\left[\varepsilon_{o}\left(1+d^{*} e^{-\eta}\right)+\sigma^{*}\left\{1-\varepsilon_{o}\left(1+d^{*} e^{-\eta}\right)\right\}\right], \\
& \gamma(\eta)=\gamma_{o}\left[\varepsilon_{o}\left(1+d^{*} e^{-\eta}\right)+\gamma^{*}\left\{1-\varepsilon_{o}\left(1+d^{*} e^{-\eta}\right)\right\}\right],
\end{aligned}
$$

For permeability, porosity, thermal conductivity and solutal diffusivity respectively.

The subscripts o for each of them are at the plate near the edge of the boundary layer. $d$ and $d^{*}$ are the terms used, fixing their values other than 0 for varying the fluid properties. If $d$ and $d^{*}$ takes 0 then the system is said to be uniform permeability (UP). This study takes $\mathrm{d}=3.0$ and $\mathrm{d}^{*}=1.5$ as a fixed value to represent the fluid properties variation, whereas the system can be called variable permeability (VP). $\sigma^{*} a n d \gamma^{*}$ are the ratios of solid to fluid of thermal conductivity and solutal diffusivity, respectively.

Substituting (6) and (7) in (2), (3), and (4) and using (8), we get,

$$
\begin{aligned}
& f^{\prime \prime \prime}+\frac{1}{2} f f^{\prime \prime}+\frac{G r}{\operatorname{Re}^{2}}(\theta-N \phi)+\frac{\alpha^{*}\left(1+d^{*} e^{-\eta}\right)}{\sigma \operatorname{Re}\left(1+d e^{-\eta}\right)}\left(1-f^{\prime}\right)-C_{\alpha} f^{(v)}+M^{2} \varepsilon_{o}\left(1+d^{*} e^{-\eta}\right)\left(1-f^{\prime}\right)=0 \\
& \theta^{\prime \prime}=-\frac{(1 / 2) \operatorname{Pr} \theta f+\operatorname{Pr} E f^{\prime \prime 2}+\varepsilon_{o} d^{*} e^{-\eta}\left(\sigma^{*}-1\right) \theta^{\prime}}{\varepsilon_{o}+\sigma^{*}\left(1-\varepsilon_{o}\right)+\varepsilon_{o} d^{*} e^{-\eta}\left(1-\sigma^{*}\right)}
\end{aligned}
$$


$\phi^{\prime \prime}=-\frac{(1 / 2) S c \phi^{\prime} f+\varepsilon_{o} d^{*} e^{-\eta}\left(\gamma^{*}-1\right) \phi^{\prime}}{\varepsilon_{o}+\gamma^{*}\left(1-\varepsilon_{o}\right)+\varepsilon_{o} d^{*} e^{-\eta}\left(1-\gamma^{*}\right)}$,

(9), (10), and (11) as shown above, where, $\operatorname{Pr}=\bar{\mu} / \rho \alpha_{o}$ is the Prandtl number, $S c=\bar{\mu} / \rho \gamma_{o}$ is the Schmidt number, $\alpha^{*}=\mu / \bar{\mu}$ is the ratio of viscosities, $N=\frac{\beta_{C}\left(C_{w}-C_{\infty}\right)}{\beta_{T}\left(T_{w}-T_{\infty}\right)}$ is the Buoyancy ratio, $E=U_{o}^{2} / C_{p}\left(T_{w}-T_{\infty}\right)$ is the Eckert number, $\sigma=k_{o} / x^{2} \varepsilon_{o}$ is the local permeability parameter, $\quad \operatorname{Re}=U_{o} x / v$ is the local Reynolds number and $G r_{T o r C}=g \beta_{T o r C}\left(T_{W}-T_{\infty}\right) x^{3} / v^{2}$ is the thermal or solutal Grashof number, $C_{\alpha}=\frac{\mu_{c}}{\mu \mathrm{x}^{2}} \operatorname{Re}$ is the couple stress parameter, $G r / \operatorname{Re}^{2}$ mixed convection parameter and $M^{2}=\frac{\sigma_{m} B_{0}^{2} x}{\left(\rho U_{0}\right)}$ is the magnetic field parameter. Thermal and Solutal Grashof numbers are the same.

The Boundary conditions (5) are now transformed to:

$f=0, \quad f^{\prime}=0, \quad f^{\prime \prime}=0, \quad \theta=1, \quad \phi=1 \quad$ at $\quad \eta=0$,

$f^{\prime}=1, \quad f^{\prime \prime}=0, \quad \theta=0, \quad \phi=0 \quad$ as $\eta \rightarrow \infty$.

Apart from solving the basic equations existing for the physical system, practically applicable terms to be calculated, which leads to heat and mass transport. They can be expressed as:

$\tau=-f^{\prime \prime}(0) / \sqrt{\operatorname{Re}}, \quad N u=-\sqrt{\operatorname{Re}} \theta^{\prime}(0)$ and $S h=-\sqrt{\operatorname{Re}} \phi^{\prime}(0)$

where $\mathrm{Nu}$ and Sh are the Nusselt and Sherwood numbers, respectively and is the skin friction.

\subsection{Method of solution.}

The boundary value problem equations obtained from non-linear equations are in (9) (11). They are highly coupled and are of higher-order, difficult to solve. The choice of values initially does not match the solution at the end of the plate when we apply RK method of $4^{\text {th }}$ order explicitly. Hence, we convert the higher-order to first-order equations starting with $f_{1}=f, f_{6}=\theta, f_{8}=\phi$, the derivative being with respect to which are expressed as follows:

$\frac{d f_{5}}{d \eta}=\frac{1}{C_{\alpha}}\left[\frac{1}{2} f_{1} f_{3}+\frac{G r}{\operatorname{Re}^{2}}\left(f_{6}-N f_{8}\right)+f_{4}-\frac{\alpha^{*}}{\sigma \operatorname{Re}}\left(\frac{1+d^{*} e^{-\eta}}{1+d e^{-\eta}}\right)\left(1-f_{2}\right)+M^{2} \varepsilon_{o}\left(1+d^{*} e^{-\eta}\right)\left(1-f_{2}\right)\right]$

$\frac{d f_{7}}{d \eta}=-\frac{(1 / 2) \operatorname{Pr} f_{1} f_{7}+\operatorname{Pr} E f_{3}^{2}+\varepsilon_{o} d^{*} e^{-\eta}\left(\sigma^{*}-1\right) f_{7}}{\varepsilon_{o}+\sigma^{*}\left(1-\varepsilon_{o}\right)+\varepsilon_{o} d^{*} e^{-\eta}\left(1-\sigma^{*}\right)}$,

$\frac{d f_{9}}{d \eta}=-\frac{(1 / 2) S c f_{1} f_{9}+\varepsilon_{o} d^{*} e^{-\eta}\left(\gamma^{*}-1\right) f_{9}}{\varepsilon_{o}+\gamma^{*}\left(1-\varepsilon_{o}\right)+\varepsilon_{o} d^{*} e^{-\eta}\left(1-\gamma^{*}\right)}$,

According the boundary conditions takes the form

$f_{1}(0)=0, f_{2}(0)=0, f_{3}(0)=0, f_{6}(0)=1, f_{8}(0)=1$

$f_{2}(\infty)=1, f_{3}(\infty)=1, f_{6}(\infty)=0, f_{8}(\infty)=0$.

The system of differential equations (15) is of the first order. As it says to solve them, 9 initial conditions are needed. However, only five initial conditions ( 3 on $f, 1$ on $\theta$, and 1 on 
$\phi$ ) are available. The other four conditions are found with the help of boundary conditions

(17) at the end of the boundary far away from the plate. A technique where we guess the finite value at far away from the plate called as shooting technique is employed here. This guess of appropriate initial finite value should be accurate depending on the convergence obtained. When compared with the dependent variable's calculated values far away from the plate with the value at the given point, the guess value accuracy can be checked with the understanding that no differences appear. If the difference is observed, the above process is repeated until we obtain the improvised values and terminate when the value reaches 10-6, thus the converged target results are obtained. The accuracy with the above process can be obtained using the RK method of fifth-order accuracy compared to some authors in the literature survey who have used the fourth-order accuracy. Mohammadein and El-Shaer [13] used RK method with only fourth-order accuracy who initiated varying the permeability for heat transfer study.

\section{Results and Discussion}

As discussed above in the solution method, A technique called the shooting method is employed to the I order equations (15) with RK Fehlberg followed by NR method. Table 1 shows the values tabulated with certain fixed values and varying values.

\subsection{Effect of Hartmann number (M).}

In Figure. 2 and 3, as observed for the fixed couple stress $C_{\alpha}=1.0$ and varying the magnetic parameter M in VP case leads to an increase in the boundary layer of velocity and decrease in temperature, which is due to accelerating force from magnetic field leading to reduction of the frictional resistance. The reduction in frictional resistance depends on M's numerical quantity, where it's force suppresses the buoyancy effect, which is mainly responsible for interactions occurred in velocity and temperature spread over. For fixed $C_{\alpha}=$ 1.0 and $M=1$ the velocity and concentration distributions are shown in Figs. 4 and 5 for VP case by varying the values of $\mathrm{N}$. The velocity boundary layer rises when the buoyancy ratio rises.

\subsection{Effect of Couple Stress Parameter $\left(C_{\alpha}\right)$.}

The additive fluid considered in the study generates the rotational field of the velocity. The high velocity reduces in amplitude and the direction moves to the far region away from the vertical heated plate. This additive fluid, when increased, the effect of a decrease in the velocity boundary layer is shown in Fig. 6. Nevertheless, the increase in the parameter leads to an increase in temperature and an increase in the concentration distributions. Fluid generating the rotational field is the main key to the effects observed in Figs. 7 and 8. The energy and the solute for their transfer identify with being more than the fluid being used to be Newtonian.

\subsection{Effect of mixed convection parameter.}

The Grashof number ratio to the square of Reynolds number represents the mixed convection parameter, which is mainly responsible for heat and mass transfer (convection). Variations of this parameter with the addition of magnetic parameter and couple stress and fluid are considered, and observations are shown in the Figs. 9 -11. A rise in this ratio leads to a rise in the induced flow. This induced flow increases the mean velocity in a vertical 
direction. Hence, as shown in the physical system, the vertical heated plate placed along the $\mathrm{x}$ direction and velocity in an upward direction, the heat transfer currents are transferred from the $\mathrm{x}$-axis where the plate is fixed to the infinite region gives rise to fluid velocity, which increases when the ratio increases as it affects directly to the mean velocity. These parameter effects are observed and compared for the constant fluid properties and varying them, i.e., constant and variable cases. The latter case shows the effect to be more prominent than the former. Similar effects are observed for the boundary layer of temperature and concentration for the increase in the mixed convection parameter ratio both in UP and VP cases.

Table 1. Results for $f^{\prime \prime}(0),-\theta^{\prime}(0)$ and - $\phi^{\prime}(0)$ for Uniform Permeability (UP) and Variable Permeability

\begin{tabular}{|c|c|c|c|c|c|c|c|c|c|c|c|}
\hline \multirow[b]{2}{*}{$C_{\alpha}$} & \multirow[b]{2}{*}{$\mathbf{M}$} & \multirow[b]{2}{*}{$\mathbf{N}$} & \multirow{2}{*}{$\sigma^{*}$} & \multirow{2}{*}{$\mathrm{Gr} / \mathrm{Re}^{2}$} & \multirow[b]{2}{*}{$\alpha^{*} / \sigma \operatorname{Re}$} & \multicolumn{3}{|c|}{$\begin{array}{l}(\mathrm{VP}) \text { cases. } \\
\text { Uniform Permeability (UP) }\end{array}$} & \multicolumn{3}{|c|}{ Variable Permeability (VP) } \\
\hline & & & & & & $f^{\prime \prime}(0)$ & $-\theta^{\prime}(0)$ & $-\phi^{\prime}(0)$ & $f^{\prime \prime}(0)$ & $-\theta^{\prime}(0)$ & $-\phi^{\prime}(0)$ \\
\hline \multirow{14}{*}{1.0} & \multirow{10}{*}{0.0} & \multirow{10}{*}{1.0} & \multirow{8}{*}{2.0} & \multirow{4}{*}{0.2} & 0.0 & 0.453210 & 0.351230 & 0.341110 & 0.500345 & 0.361780 & 0.350020 \\
\hline & & & & & 0.1 & 0.356784 & 0.281765 & 0.271245 & 0.371650 & 0.321340 & 0.311450 \\
\hline & & & & & 0.2 & 0.486750 & 0.381560 & 0.361450 & 0.500323 & 0.425678 & 0.400345 \\
\hline & & & & & 0.5 & 0.881340 & 0.451650 & 0.441030 & 0.900544 & 0.481954 & 0.461345 \\
\hline & & & & 0.0 & \multirow{4}{*}{0.1} & 0.443210 & 0.381340 & 0.373450 & 0.463450 & 0.402563 & 0.391786 \\
\hline & & & & 0.2 & & 0.356784 & 0.251678 & 0.281765 & 0.371650 & 0.321340 & 0.311450 \\
\hline & & & & 1.0 & & 0.754320 & 0.481230 & 0.472310 & 0.773567 & 0.502345 & 0.489876 \\
\hline & & & & 2.0 & & 0.897650 & 0.564320 & 0.551120 & 0.924567 & 0.594322 & 0.581567 \\
\hline & & & 4.0 & \multirow{2}{*}{0.2} & \multirow{2}{*}{0.1} & 0.551430 & 0.381450 & 0.371110 & 0.554780 & 0.435670 & 0.430020 \\
\hline & & & 6.0 & & & 0.651780 & 0.423130 & 0.411110 & 0.661760 & 0.451340 & 0.440230 \\
\hline & \multirow{4}{*}{1.0} & 0.0 & \multirow{4}{*}{2} & \multirow{4}{*}{0.2} & \multirow{4}{*}{0.1} & 0.356430 & 0.287650 & 0.271560 & 0.451320 & 0.321560 & 0.310050 \\
\hline & & 1.0 & & & & 0.356784 & 0.251678 & 0.243765 & 0.881650 & 0.421560 & 0.420030 \\
\hline & & 5.0 & & & & 0.987650 & 0.657867 & 0.641230 & 0.987060 & 0.564530 & 0.551003 \\
\hline & & 10.0 & & & & 0.998760 & 0.682430 & 0.671220 & 0.999340 & 0.657890 & 0.641560 \\
\hline 0.0 & \multirow{3}{*}{5.0} & \multirow{5}{*}{1.0} & \multirow{5}{*}{2.0} & \multirow{5}{*}{0.2} & \multirow{5}{*}{0.1} & 0.674535 & 0.567430 & 0.572340 & 0.773567 & 0.591678 & 0.581678 \\
\hline 0.5 & & & & & & 0.656784 & 0.351423 & 0.341110 & 0.683220 & 0.356750 & 0.350120 \\
\hline 1.0 & & & & & & 0.356784 & 0.281765 & 0.271245 & 0.371650 & 0.321340 & 0.311450 \\
\hline 1.5 & \multirow{2}{*}{10.0} & & & & & 0.451320 & 0.371540 & 0.370020 & 0.519430 & 0.426510 & 0.420110 \\
\hline 2.0 & & & & & & 0.551230 & 0.391030 & 0.381230 & 0.647890 & 0.456130 & 0.450020 \\
\hline
\end{tabular}

3.4. Effect of the ratio $\left(\alpha^{*} / \sigma R e\right)$.

Increasing values in the ratio $\alpha^{*} / \sigma$ Releads velocity to increase observed in Fig. 12, but leads to decrease in energy and solute profiles shown in Figs. 13 and 14. In all the above three profiles, the effect is due to a very low Reynolds number giving the high viscous force. A moderate change of observations is seen in both the cases of uniform permeability and variable permeability.

\subsection{Effect of Prandtl number (Pr) and Schmidt number (Sc).}

Figure.15 and 16 are plotted for different Prandtl numbers 0.71, 3.0, and 7.0. Prandtl number defines to be the ratio of momentum to thermal diffusivities; heat is transferred from the heated plate as its value increases, leading to a rise in the boundary layer of velocity and a decrease in the thermal boundary layer thickness. The average temperature is lowered, and 
similar behavior but not very prominent is observed with the Schmidt number Sc increased value for concentration distributions observed in Fig. 17.

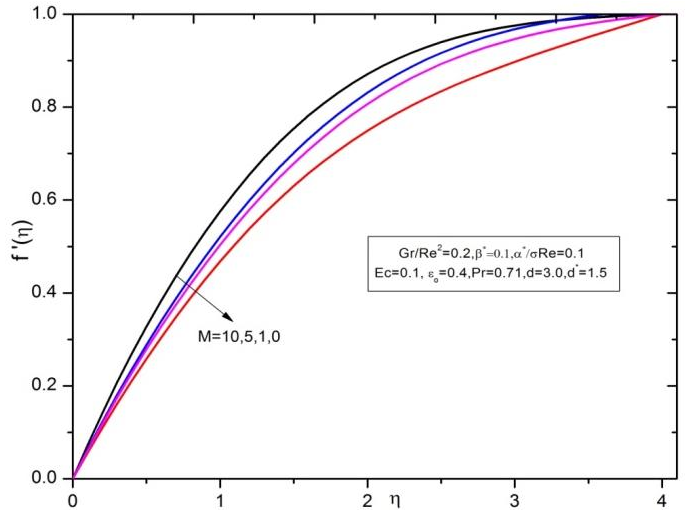

Figure 2. VP case for Velocity graphs varying M and fixed $\mathrm{C} \alpha$.

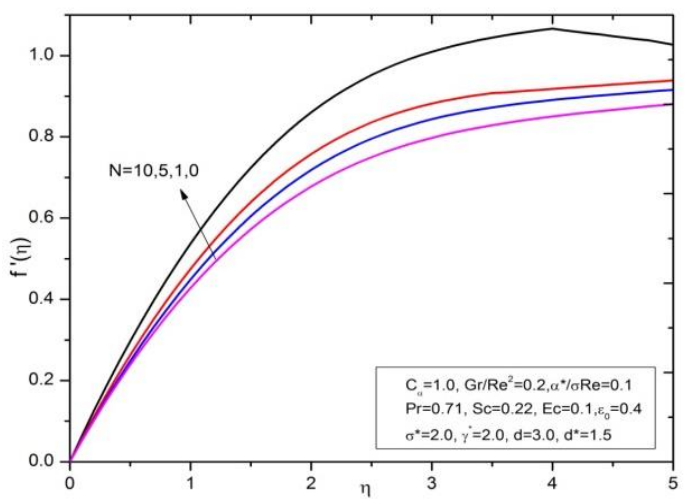

Figure 4. VP case Velocity graphs different Values of $\mathrm{N}$.

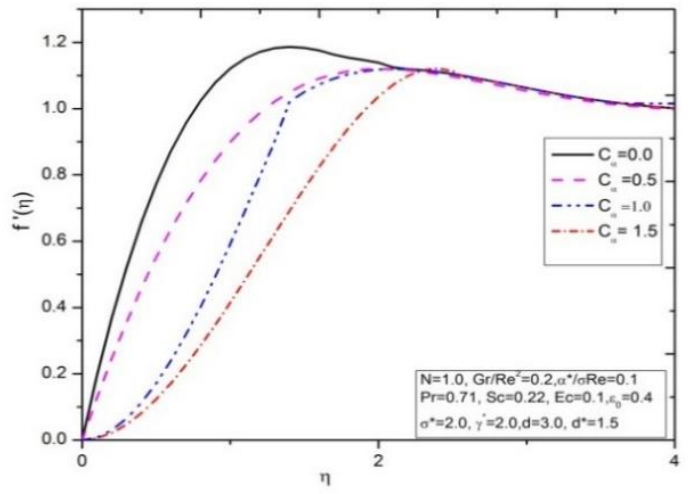

Figure 6. Velocity graphs for $C_{\alpha}$ values in VP case.

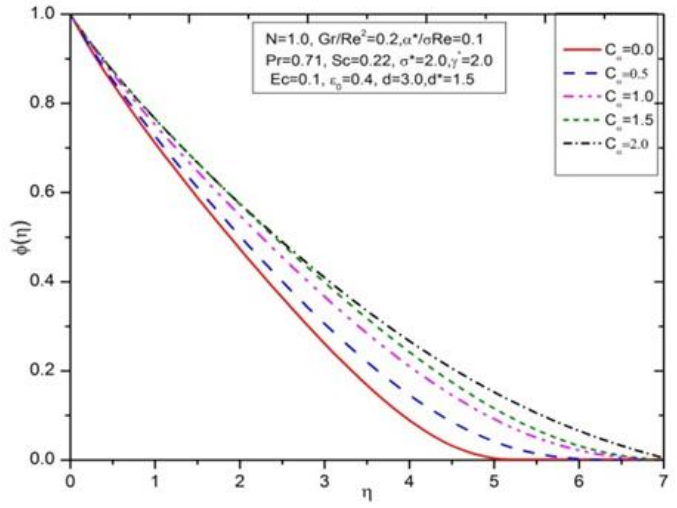

Figure 8. VP case conversation variation for different $C_{\alpha}$.

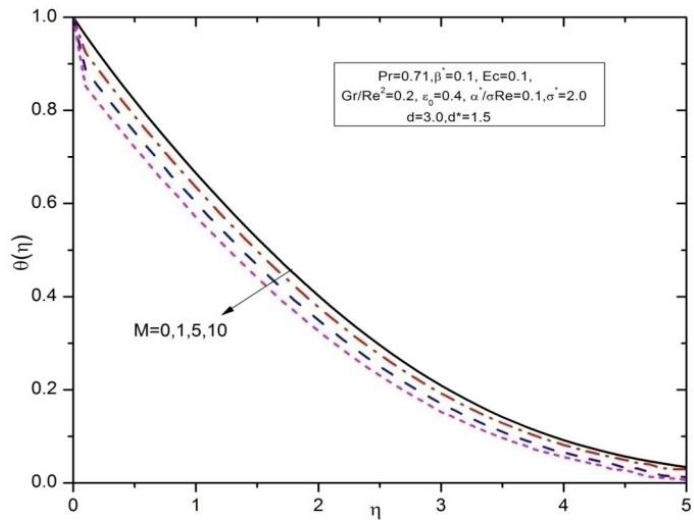

Figure 3. VP case for temperature Graphs for varying $\mathrm{M}$ with fixed $C_{\alpha}$.

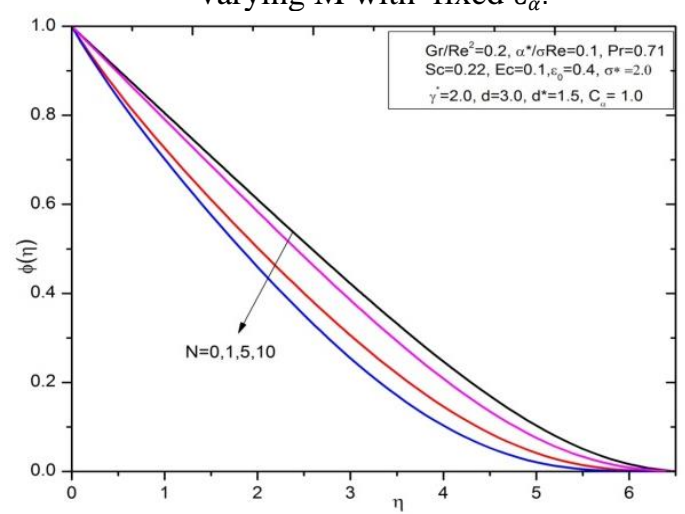

Figure 5. VP case concentration graphs for different $\mathrm{N}$.

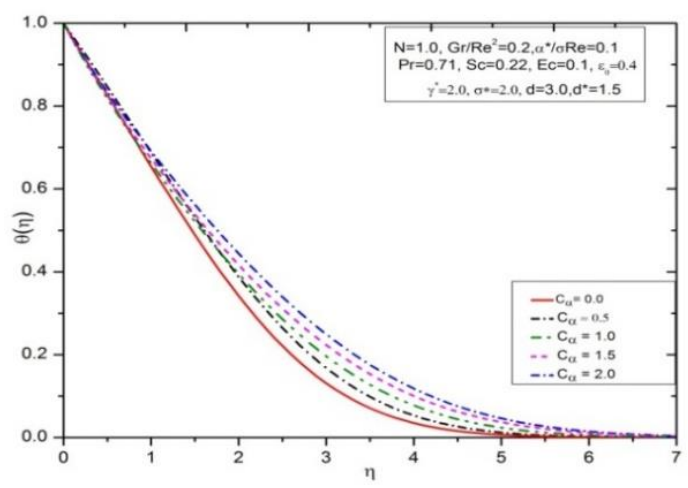

Figure 7. VP case temparature graphs for different $C_{\alpha}$.

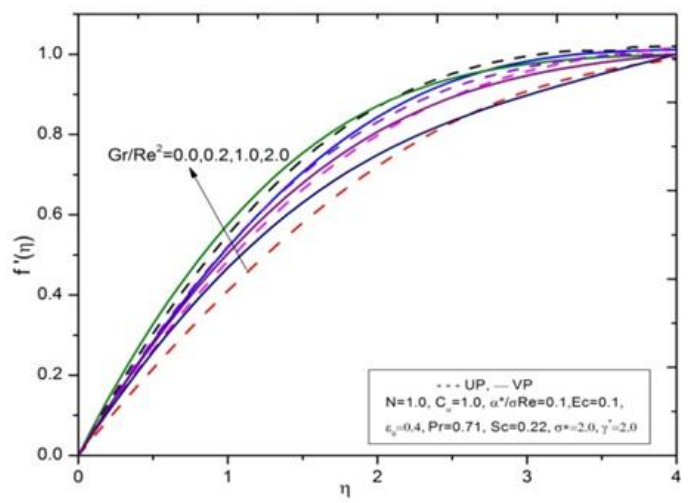

Figure 9. $\mathrm{Gr} / \mathrm{R} e^{2}$ variations for velocity distributions. 


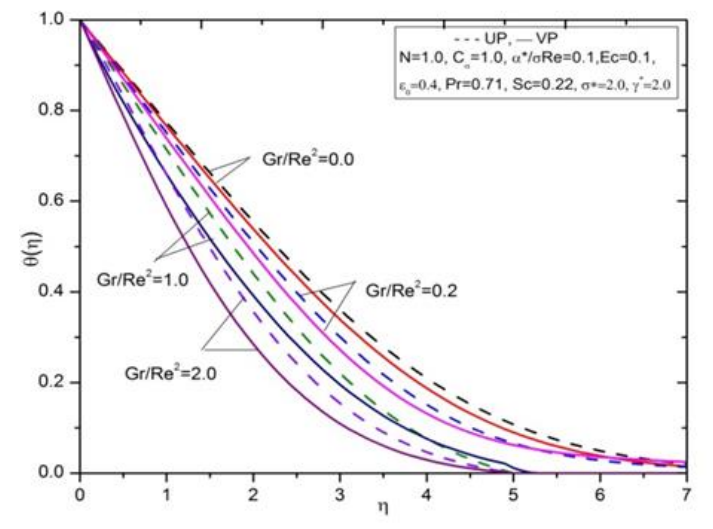

Figure 10. $G r / R e^{2}$ variations for temperature distribution.

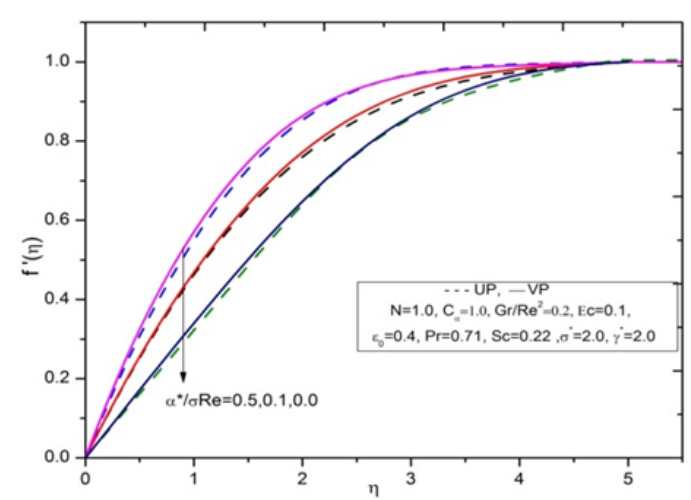

Figure 12.Velocity graphs for different values of $\alpha^{*} / \sigma \operatorname{Re}$.

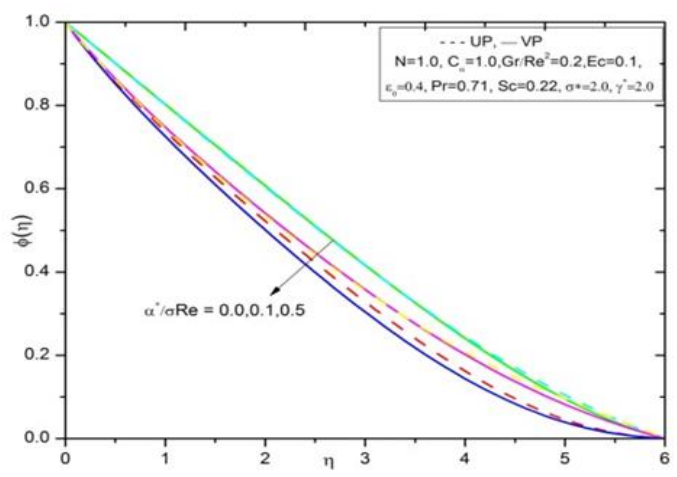

Figure 14.Concentration graphs for Various $\alpha^{*} / \sigma \operatorname{Re}$.

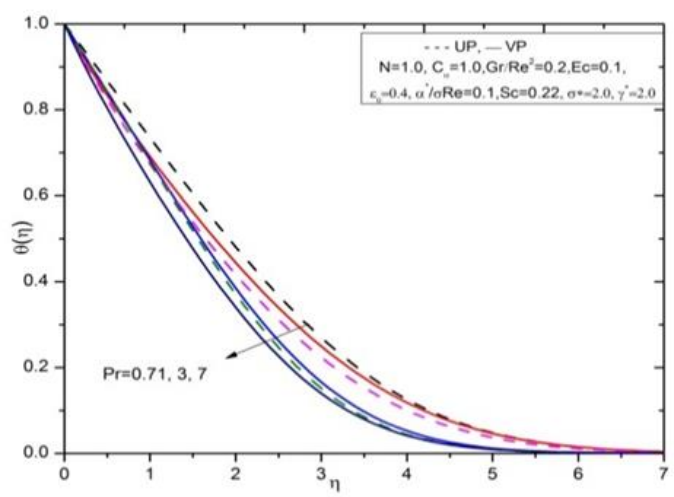

Figure 16.Temperature graphs for different values of Pr.

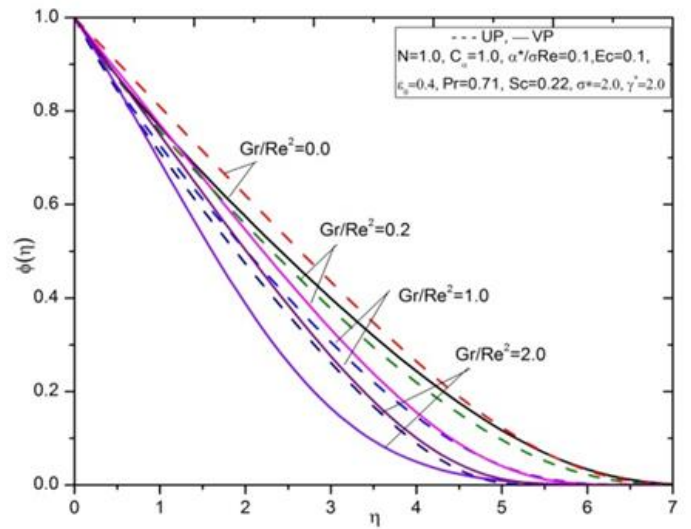

Figure 11. $G r / R e^{2}$ variations for concentration distributions.

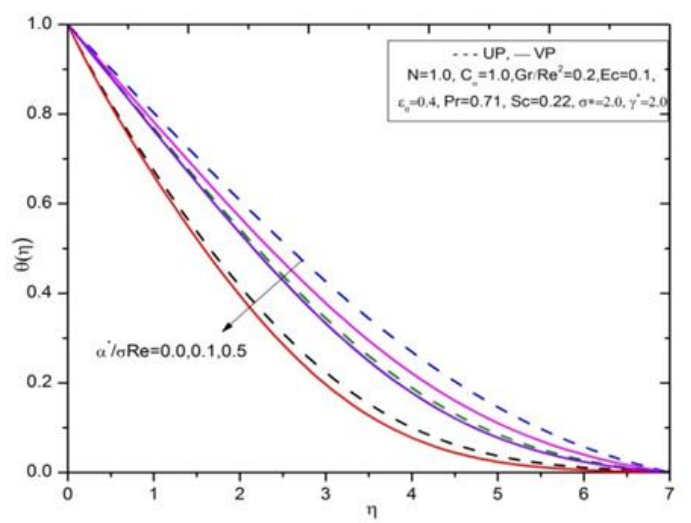

Figure 13. Temperature graphs for different $\alpha^{*} / \sigma \operatorname{Re}$.

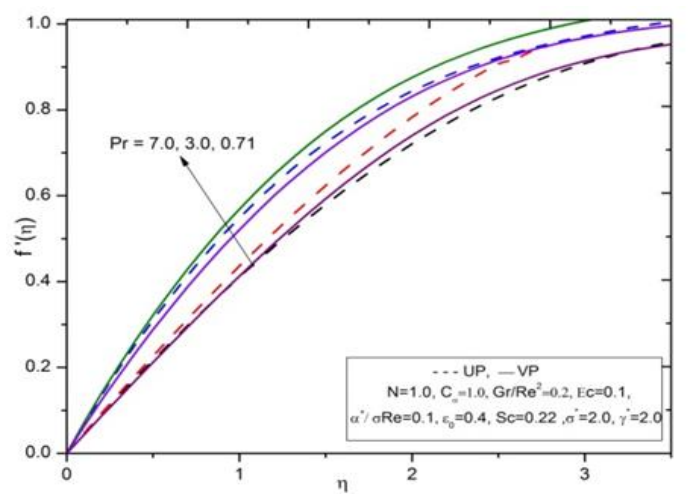

Figure 15. Velocity graphs for different values of Pr.

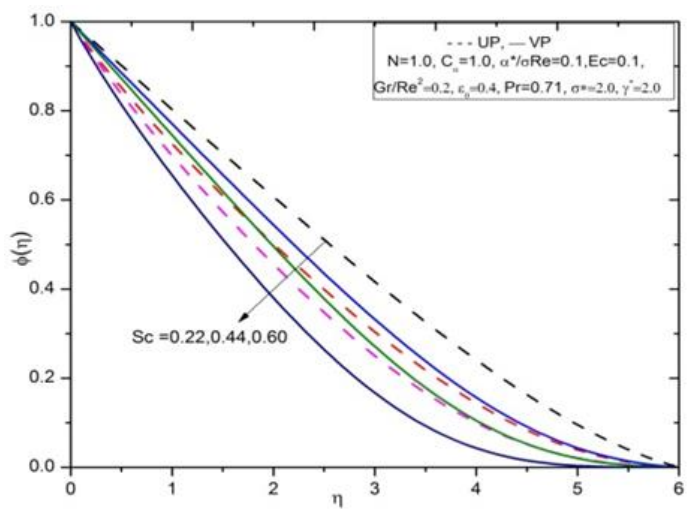

Figure 17. Concentration graphs for different values of Sc. 


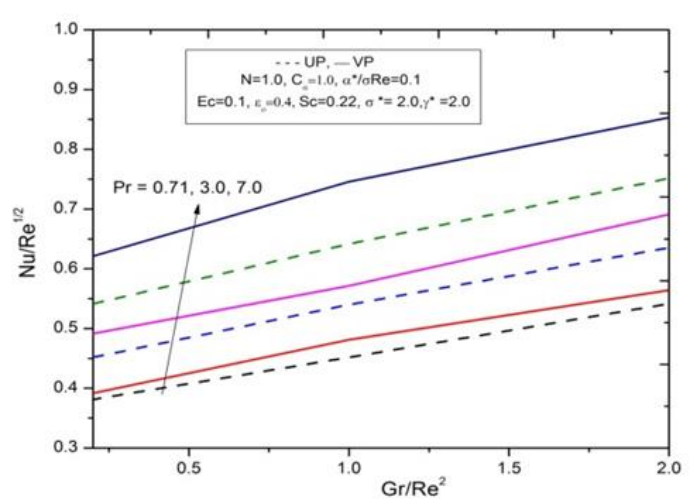

Figure 18.UP and VP cases for different Pr with Variations of $\mathrm{Nu}$ versus $\mathrm{Gr} / \mathrm{Re}^{2}$.

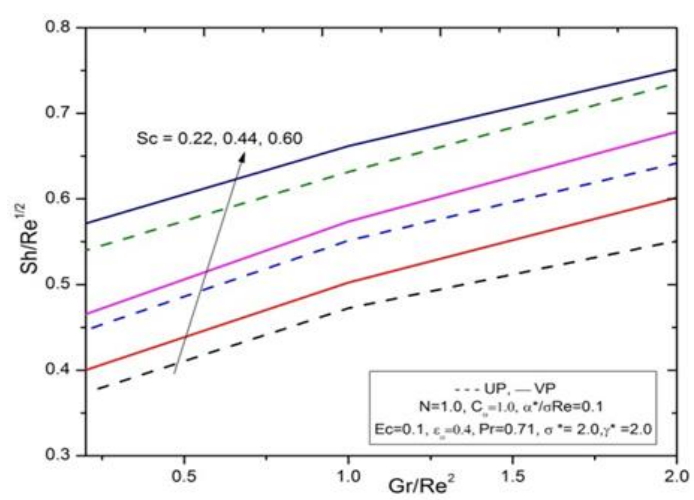

Figure 19. UP and VP cases for Sc with variations of Sh versus $G r / \mathrm{Re}^{2}$.

\subsection{Effect of Nusselt number $(\mathrm{Nu})$.}

The heat transfer coefficient $\mathrm{Nu}$ versus the mixed convection parameter for different Prandtl number Pr values is shown in Fig. 18. It is observed that there is a linear increase of heat transfer for Pr range values of $0.71,3$, and 7.0 in both UP and VP cases. A close observation explains that the variable permeability case is faster and more dominant compared to the constant fluid property case.

\subsection{Effect of Sherwood number (Sh).}

The mass transfer coefficient Sh versus the mixed convection parameter for Schmidt number Sc's different values is shown in Fig. 19. It is observed that there is a linear increase of mass transfer for Sc range values 0.22, 0.44 and 0.60 in both UP and VP cases. A close observation leads to understanding that VP case is more dominant compared to UP case.

\section{Conclusions}

A numerical model is developed for double-diffusive mixed convection heat and mass transfer over a vertical heated plate in the presence of magnetic field and couple stress fluids by considering the variable fluid properties like permeability, porosity, thermal conductivity, and solutal diffusivity. The Governing equations are highly coupled and non-linear in nature. They are transformed into ordinary differential equations and further to the first-order ODE using similarity variables. The obtained equations are solved numerically by using the shooting method. The computed results are represented graphically to illustrate the heat and mass transfer characteristics of the physical parameters in consideration. The following conclusions are drawn: increase in the magnetic parameter $M$ increases the velocity distributions and decreases the temperature distributions for variable permeability (VP) case from the plate to the free stream; increase in the second-order resistance increases the velocity profiles and decreases the temperature profiles. The boundary layer rises near the plate and asymptotes towards the free stream; an increase in couple stress parameter decreases the maximum velocity in amplitude and the location of the maximum velocity moves far away from the wall due to the rotational field of the velocity generated in couple stress fluid for variable permeability case; this observation is due to the reciprocal of the couple stress parameter multiplied by the momentum equation; temperature and concentration of the fluid increases with the increase of couple stress fluid parameter; the mixed convection parameter variations show how convection currents are carried away and affect the velocity, temperature, and concentration distributions; 
lower the Prandtl number Pr, the higher the velocity overshoot and the lesser in the thermal boundary layer. Higher the Schmidt number, the concentration profiles decreases; the local Nusselt number and local Sherwood number with variations of the mixed convection parameter as the Prandtl number and Schmidt number y, respectively, resulting in the increasing behavior for higher values of Prandtl and Schmidt number.

\section{Funding}

This research received no external funding.

\section{Acknowledgments}

The Researchers here are very grateful to the Research Centers of Atria Institute of Technology, Ramaiah Institute of Technology (affiliated to VTU) for their extended support.

\section{Conflicts of Interest}

The authors declare no conflict of interest.

\section{References}

1. Stokes, V.K. Couple Stresses in Fluids. The Physics of Fluids 1966, 9, 1709-1715, https://doi.org/10.1063/1.1761925.

2. Walicki, E.; Walicka, A. Inertia and couple-stress effects on squeeze-film characteristics with reference to biological bearings. Tribotest 2002, 8, 195-203, http://dx.doi.org/10.1002/tt.3020080302.

3. Devakar, M; Iyengar,T. K. V.; Stokes' Problems for an Incompressible Couple Stress Fluid. Non-linear Analysis: Modelling and Control2008, 13, 181-190,http://dx.doi.org/10.15388/NA.2008.13.2.14578.

4. Farooq, M.; Islam, S.; Rahim, M.T.; Siddiqui, A. Laminar flow of couple stress fluids for Vogel's model. Scientific research and essays 2012, 7, 2936-2961.

5. Mobolaji, H.O.; Akpobi, J.A. Effects of Couple Stress Lubricants on Pressure and Load Capacity of Infinitely Wide Exponentially Shaped Slider Bearing. Proceedings of the World Congress on Engineering and Computer Science. WCECS 2010, San Francisco,USA, Volume 2, October 20-22, 2010.

6. Mishra, N.; Sharma, V. Effect of mass transfer on the free convective flow through a porous medium with variable permeability in slip flow regime with couple stresses. American Journal of Scientific and Industrial Research 2011, 2, 469-477,https://doi.org/10.5251/ajsir.2011.2.3.469.477.

7. Mohammadein, A.A.; El-Shaer, N.A. Influence of variable permeability on combined free and forced convection flow past a semi-infinite vertical plate in a saturated porous medium. Heat and Mass Transfer 2004, 40, 341-346,https://doi.org/10.1007/s00231-003-0430-3.

8. Nalinakshi, N.; Dinesh, P.; Chandrashekar, D.J.I.j.o.e.r.; technology. Numerical Study of Double Diffusive Mixed Convection with Variable Fluid Properties.International Journal of Engineering Research and technology 2,2013, 131-139.

9. Dinesh, P.A.; Nalinakshi, N.; Sandeep, N. Double diffusive mixed convection in a couple stress fluids with variable fluid properties. Advances in physics theories and applications 2015, 41, 30-42

10. Suresh Babu, R.; Kumar, B.R.; Dinesh, P.A. Effects of Mixed Convection on the Oscillatory Flow of a Couple Stress Fluid through a Vertical Plate with Variable Fluid Properties. Defect and Diffusion Forum 2018, 388, 328-343,https://doi.org/10.4028/www.scientific.net/DDF.388.328.

11. Uma, M.; Dinesh, P.A.; Vasudevamurthy, A.S. Effects of Forchheimer, MHD and Radiation Absorption for Chemically Reacting Unsteady Dusty Viscoelastic Fluid Couette Flow in an Irregular Channel.In: Advances in Fluid Dynamics, Lecture Notes in Mechanical Engineering.2020; pp. 9991012,https://doi.org/10.1007/978-981-15-4308-1_77.

12. Nalinakshi, N.; Dinesh, P. A. Thermo-diffusion and diffusion-thermo effects for a Forchheimer model with MHD over a vertical heated plate. In: Advances in Fluid Dynamics(LNME).2020; 343361,https://doi.org/10.1007/978-981-15-4308-1_27.

13. Suma, S.P.; Sudhir, P.; Ramesh, N.L.; Dinesh, P.A. Characteristic Study of Coriolis Force on Free Convection in a Finite Geometry with Isotropic and Anisotropic Porous Media In: Advances in Fluid Dynamics, Lecture Notes in Mechanical Engineering.2020;pp. 985-998, https://doi.org/10.1007/978-98115-4308-1_76. 
14. Shilpa, B.V.; Chandrashekar, D.V.; Dinesh, P.A.; Eswara, A.T.Analytical approach for mixed convective flow in presence of Casson fluid in a Porous channel. In:Advances in Fluid Dynamics, Lecture Notes in Mechanical Engineering.2020;pp. 939-952,https://doi.org/10.1007/978-981-15-4308-1_71.

15. Gangadhar, K.; Kannan, T.; DasaradhaRamaiah, K.; Sakthivel, G. Boundary layer flow of nano-fluids to analyse the heat absorption/generation over a stretching sheet with variable suction/injection in the presence of viscous dissipation. International Journal of Ambient Energy 2020, 41, 969-980, https://doi.org/10.1080/01430750.2018.1501738.

16. Srinivasacharya, D.; Jagadeeshwar, P. Effect of Joule heating on the flow over an exponentially stretching sheet with convective thermal condition. Mathematical Sciences 2019, 13, 201211,https://doi.org/10.1007/s40096-019-0290-8.

17. Double Diffusive Convection and Internal Heat Generation with Soret and Dufour Effects over an Accelerating Surface with Variable Viscosity and Permeability.Advances in Physics Theories and Application 2018, 69.

18. Chu, X.; Yang, G.; Pandey, S.; Weigand, B. Direct numerical simulation of convective heat transfer in porous media. International Journal of Heat and Mass Transfer 2019, 133, 11-20, https://doi.org/10.1016/j.ijheatmasstransfer.2018.11.172.

19. Syam Sundar, L.; Ravi Kumar, N.T.; Addis, B.M.; Bhramara, P.; Singh, M.K.; Sousa, A.C.M. Heat transfer and effectiveness experimentally-based analysis of wire coil with core-rod inserted in $\mathrm{Fe} 3 \mathrm{O} 4 /$ water nanofluid flow in a double pipe U-bend heat exchanger. International Journal of Heat and Mass Transfer 2019, 134, 405-419, https://doi.org/10.1016/j.ijheatmasstransfer.2019.01.041.

20. Idowu, A.S.; Falodun, B.O. Soret-Dufour effects on MHD heat and mass transfer of Walter's-B viscoelastic fluid over a semi-infinite vertical plate: spectral relaxation analysis. Journal of Taibah University for Science 2019, 13, 49-62, https://doi.org/10.1080/16583655.2018.1523527.

21. Irfan, M.; Khan, M.; Khan, W.A. Impact of non-uniform heat sink/source and convective condition in radiative heat transfer to Oldroyd-B nano-fluid: A revised proposed relation. Physics Letters A 2019, 383, 376-382, https://doi.org/10.1016/j.physleta.2018.10.040.

22. Ahmed, J.; Khan, M.; Ahmad, L. Significance of thermophoresis, thermal-diffusion and diffusion-thermo on the flow of Maxwell liquid film over a horizontal rotating disk. Physica Scripta 2019, 94,https://doi.org/10.1088/1402-4896/ab120d.

23. Sravan Kumar, T.; Dinesh, P.A.; Makinde, O.D. Impact of Lorentz Force and Viscous Dissipation on Unsteady Nanofluid Convection Flow over an Exponentially Moving Vertical Plate. Mathematical Models and Computer Simulations 2020, 12, 631-646, https://doi.org/10.1134/S2070048220040110.

24. Shreeedhar, G; Rama, B.R. Chemical reaction effect on unsteady MHD flow past an infinite vertical porous plate in the presence of heat absorption, International Journal of Advanced Research in Engineering and Technology2019, 10, 95-103,https://doi.org/10.34218/IJARET.10.1.2019.010.

25. Ayegbusi, F.D.; Onwubuoya, C.; Falodun, B.O. Unsteady problem of magnetohydrodynamic heat plus mass transfer convective flow over a moveable plate with effects of thermophoresis and thermal radiation. Heat Transfer 2020, 49, 3593-3612,https://doi.org/10.1002/htj.21790.

26. Kempannagari, A.K.; Buruju, R.R.; Naramgari, S.; Vangala, S. Effect of Joule heating on MHD nonNewtonian fluid flow past an exponentially stretching curved surface. Heat Transfer 2020, 49, 3575-3592, https://doi.org/10.1002/htj.21789.

27. Krishna, M.V.; Swarnalathamma, B.V.; Chamkha, A.J. Investigations of Soret, Joule and Hall effects on MHD rotating mixed convective flow past an infinite vertical porous plate. Journal of Ocean Engineering and Science 2019, 4, 263-275, https://doi.org/10.1016/j.joes.2019.05.002.

28. Dinesh, P.A.; Vasudevamurthy, A.S.; Uma, M. Effects of Forchheimer, MHD and radiation adsorption for chemically reacting unsteady dusty viscoelastic fluid Couette flow in an irregular channel. In: Advances in Fluid Dynamics (LNME).2020;pp. 999-1012,https://doi.org/10.1007/978-981-15-4308-1_77.

29. Shankar, G.B.; Suresh, B.; Raja, S.M.N.; Srinivasa, G. Mass Transfer Effects on MHD Flow through Porous Medium past an Exponentially Accelerated Inclined Plate with Variable Temperature and Thermal Radiation.International journal of thermos fluid science and technology2019, 6, 114,https://doi.org/10.36963/IJTST.19060402.

30. Uma, M.; Dinesh, P.A.; Girinath, R.M.; Sreevallabha, R.A. Combined effects of Forchheimer, Soret and Dufour on MHD mixed convective dusty viscoelastic Couette flow in an irregular channel. Journal of Multidiscipline in Modelling and Structures 2020, 17,https://doi.org/10.1108/MMMS-12-2019-0233.

31. Abdal, S.; Ali, B.; Younas, S.; Ali, L.; Mariam, A. Thermo-Diffusion and Multislip Effects on MHD Mixed Convection Unsteady Flow of Micropolar Nanofluid over a Shrinking/Stretching Sheet with Radiation in the Presence of Heat Source. Symmetry 2020, 12,http://doi.org/10.3390/sym12010049.

32. Basavaraj,M.S.; Girinath Reddy, M.; Aruna, A.S.; Dinesh P.A. A non-linear mixed convective oscillatory flow over a semi-infinite vertical plate through porous medium under uniform magnetic field. Int.Journal of Advanced Research 2020, 8, 308-321,https://dx.doi.org/10.21474/IJAR01/11100.

33. Filahi, I.; Bourich, M.; Hasnaoui, M.; Amahmid, A. Analytical and Numerical Study of Soret and Dufour Effects on Thermosolutal Convection in a Horizontal Brinkman Porous Layer with a Stress-Free Upper Boundary. Mathematical Problems in Engineering 2020, 2020, 1-17, https://doi.org/10.1155/2020/4046570. 